\title{
Mit Wells-Score und D-Dimer-Test Lungenembolien ausschließen
}

- Nur $10-15 \%$ der Patienten, die aufgrund des Verdachts auf eine Lungenembolie stationär eingewiesen werden, haben tatsächlich ein Gerinnsel in der Lunge. Niederländische Forscher haben nun eine Vorgehensweise getestet, mit der sich Lungenembolien auch in einer Hausarztpraxis zuverlässig ausschließen lassen.

In ihrer Validierungsstudie kombinierten sie den klinischen Wells-Score mit einer qualitativen D-Dimer-Bestimmung. Der Score setzt sich aus sieben Elementen zusammen, die mit Punkten belegt werden:

1. Klinische Zeichen und Symptome einer tiefen Venenthrombose (geschwollenes Bein und Schmerzen bei Palpation der tiefen Venen): 3 Punkte

2. Lungenembolie als wahrscheinlichste Diagnose: 3 Punkte

3. Herzfrequenz $>100$ pro Minute: 1,5 Punkte

4. Immobilisation für mehr als drei Tage oder operativer Eingriff in den vorange- gangenen vier Wochen: 1,5 Punkte

5. Lungenembolie oder tiefe Venenthrombose in der Anamnese: 1,5 Punkte

6. Hämoptyse: 1,0 Punkte

7. Malignom (in Behandlung, während der vergangenen sechs Monate therapiert oder palliative Situation): 1,0 Punkte.

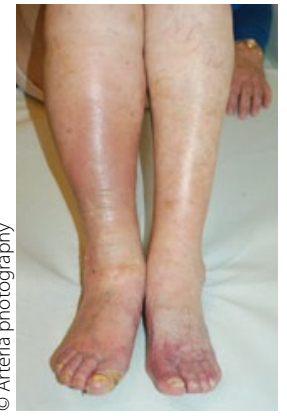

3 Punkte im WellsAls unverdächtig auf Lun- Score:Tiefe Venengenembolie gelten in der Litera- thrombose. tur Werte $\leq 4$ bzw. $<2$.

Für den D-Dimer-Nachweis wurde ein Schnelltest verwendet, der auf Konzentrationen $>80 \mathrm{ng} / \mathrm{ml}$ mit einem Farbumschlag reagiert. Nicht interpretierbare Testergebnisse wurden als positiv gewertet.

An der Studie beteiligten sich 300 Allgemeinärzte und 598 Patienten mit Verdacht auf eine Lungenembolie. Bestätigt wurde die Verdachtsdiagnose schließlich bei 73
Patienten (12,2\%). Mit der Kombination eines Wells-Scores $\leq 4$ mit einem negativen D-DimerTestergebnis wurden 272 der 598 Patienten als Gruppe mit niedrigem Risiko klassifiziert $(45,5 \%)$. Von diesen hatten vier dennoch eine Embolie - die Rate falsch negativer Ergebnisse betrug somit $1,5 \%$. Insgesamt erreichte die Sensitivität dieser Kombination einen Wert von 94,5\%. Die Spezifität lag bei $51 \%$.

„Ein Wells-Score $\leq 4$, kombiniert mit einem negativen qualitativen $D$-DimerTest, vermag eine Lungenembolie unter den Bedingungen des primären Versorgungssektors sicher und effizient auszuschließen", so das Fazit der Autoren.

DR. ROBERT BUBLAK .

Geersing GJ et al. BMJ 2012, online 4. Oktober; 345: e6564; doi: 10.1136/bmj.e6564

\section{Glosse}

\section{Männergesundheit: Kastration ist auch keine Lösung}

Männer haben es schon schwer. Weniger wegen ihres sozialen Status, obwohl dieser auch schon etwas bröckelt. Nein, es geht um ihre Gesundheit bzw. um den Zusammenhang zwischen Geschlechtshormon und Lebenserwartung.

Bei Frauen ist die Sache ja wissenschaftlich geklärt und relativ einfach: Östrogene schützen, solange sie vom Körper selbst gebildet werden, vor vielen Krankheiten, insbesondere der Arteriosklerose. Erst wenn der Körper die Hormonproduktion aufgibt und die iatrogene Zufuhr einsetzt, wird die Sache problematisch, da dies mit einigen Risiken assoziiert ist. Doch bei Männern, die lebenslang mehr oder weniger ausreichend Testosteron bilden, ist sich die Wissenschaft sehr uneinig bei der Frage: Nützt oder schadet Testosteron der Gesundheit? Auf der einen
Seite gibt es die normative Kraft des Faktischen, die klar besagt: Männer leben kürzer als Frauen und zwar genau fünf Jahre. Bisher glaubte man, entscheidend dafür sei nicht der direkte Einfluss des Testosterons auf die Manifestation bestimmter Erkrankungen, sondern die Auswirkungen des Hormons auf das Verhalten. Männer seien risikobereiter als Frauen: Mehr Risk, mehr Fun, und dafür ist man bereit, etwas früher aus dem Leben zu treten.

\section{Kastration oder Testosteron-Doping?} Studienergebnisse über Kastraten, die im 18. Jahrhundert in Südkorea lebten, legen nun nahe, dass Testosteron über den Fettstoffwechsel die Entstehung von HerzKreislauf-Erkrankungen, evtl. auch anderer Krankheiten fördern könnte; denn die Kastrierten lebten deutlich länger als Männer ohne Hypo- bzw. Agonadismus (www. springermedizin.de/3328336, MMW Nr. 17, S. 7). Männer, die angesichts dieses Befunds jetzt mit dem Gedanken an eine Kastration liebäugeln, sollten sich die Sache allerdings genau überlegen. Denn es mehren sich auch Hinweise, dass ein zu Wenig an Testosteron das metabolische Syndrom fördert und deshalb die gesundheitsfördernde Wirkung einer Kastration vollständig zu Nichte macht. Was nun, lieber Mann? Kastration oder Testosteron-Doping? Ich würde sagen: Weder noch. Bleiben Sie so, wie Sie sind! Dass die Kastration Ihnen heute wie vor 200 Jahren im alten Korea Aussicht auf ein schöneres und zugleich auch längeres Leben verspricht, wage ich nämlich zu bezweifeln.

DR. Med. Peter StiefelHAgen - 